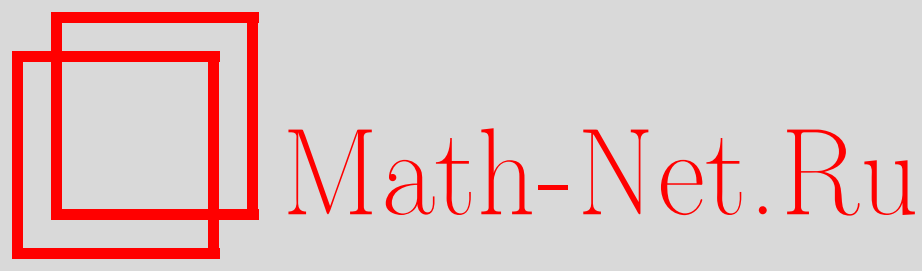

Ю. А. Трутнев, Р. М. Шагалиев, В. В. Евдокимов, А. И. Бочков, Участие В. С. Владимирова в работах по атомному проекту СССР - значимая веха в развитии основ математического моделирования процессов нейтронной физики, ТМФ, 2013, том 174, номер 2, 202-207

DOI: https://doi.org/10.4213/tmf8506

Использование Общероссийского математического портала Math-Net.Ru подразумевает, что вы прочитали и согласны с пользовательским соглашением http://www . mathnet.ru/rus/agreement

Параметры загрузки:

IP : 54.198 .64 .247

26 апреля 2023 г., 11:06:40

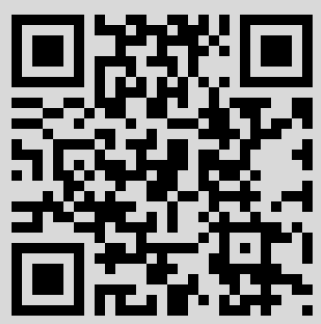




\title{
ФИЗИКА
}

Том 174, № 2

февраль, 2013

(C) 2013 г.

Ю. А. Трутнев*, Р. М. Шагалиев*,

В. В. Евдокимов*, А. И. Бочков*

\section{УЧАСТИЕ В.С. ВЛАДИМИРОВА В РАБОТАХ ПО АТОМНОМУ ПРОЕКТУ СССР - ЗНАЧИМАЯ ВЕХА В РАЗВИТИИ ОСНОВ МАТЕМАТИЧЕСКОГО МОДЕЛИРОВАНИЯ ПРОЦЕССОВ НЕЙТРОННОЙ ФИЗИКИ}

\begin{abstract}
Настоящая статья приурочена к девяностолетию со дня рождения выдающегося советского и российского ученого, академика АН СССР Василия Сергеевича Владимирова. В.С. Владимиров - один из крупнейших математиков современности, - с 1950 по 1955 г. работал в КБ-11 (сегодня Российский федеральный ядерный центр - Всероссийский научно-исследовательский институт экспериментальной физики) - на "объекте", в котором велась разработка атомного оружия. Приведены основные результаты научной деятельности В. С. Владимирова, связанные с его работой в атомном проекте СССР.
\end{abstract}

Ключевые слова: метод Владимирова, метод факторизации, вариационный принцип Владимирова, перенос нейтронов.

DOI: $10.4213 / \operatorname{tmf} 8506$

Работу над атомным проектом В.С. Владимиров начал младшим научным сотрудником Ленинградского отделения Математического института им. В. А. Стеклова. В соответствии с постановлением Совета Министров СССР по урановому проекту в мае 1948 года в ЛОМИ развернулись работы под научным руководством Л.В. Канторовича по расчетам критических масс делящегося вещества, необходимых для взрыва, и соответствующей плотности нейтронов. Типичной была задача определения радиуса шара, окруженного несколькими оболочками, при котором интегральное уравнение Пайерлса имеет положительное решение.

Испытание первой советской атомной бомбы РДС-1 было проведено 29 августа 1949 года на полигоне вблизи Семипалатинска. За свой вклад в разработку РДС-1 младший научный сотрудник В.С. Владимиров в мае 1950 года был премирован денежной суммой в размере 5000 руб.

${ }^{*}$ Российский федеральный ядерный центр - Всероссийский научно-исследовательский институт экспериментальной физики, Саров, Нижегородская обл., Россия.

E-mail: R.M.Shagaliev@vniief.ru 
В ноябре 1950 года В. С. Владимиров был переведен в КБ-11. На “объекте” он продолжил заниматься численными методами расчета критических параметров многослойных ядерных систем. Кинетическое уравнение переноса нейтронов, которое лежит в основе математических моделей для решения этого класса задач, входит в число наиболее сложных и вычислительно затратных. Очень актуальной была задача конструирования эффективных численных методов, которые были бы устойчивыми к погрешностям и позволяли бы на очень простых вычислительных устройствах того времени решать кинетическое уравнение. Понимая необходимость создания методов приближенного решения кинетического уравнения переноса нейтронов, в 1951 году В. С. Владимиров разработал оригинальный метод решения кинетических уравнений для сферически-симметричных областей, основанный на использовании характеристик интегро-дифференциального уравнения Больцмана - метод характеристик [1]-[3]. Этот метод успешно применялся для расчетов атомных и водородных зарядов. В 1951-1953 годах вручную были посчитаны десятки вариантов многослойных зарядов для водородной бомбы. За выполнение специального задания Правительства В. С. Владимирову и Н. Н. Боголюбову в декабре 1953 года была присуждена Сталинская премия СССР и присвоены звания Лауреата Сталинской премии СССР.

В литературе метод характеристик часто называют методом Владимирова [1]-[4]. Рассмотрим метод Владимирова применительно к решению кинетического уравнения для сферы:

$$
\begin{gathered}
\mu \frac{\partial \varphi(r, \mu)}{\partial r}+\frac{1-\mu^{2}}{r} \frac{\partial \varphi(r, \mu)}{\partial \mu}+\alpha(r) \varphi(r, \mu)=F(r), \\
F(r)=\frac{\beta(r)}{2} \varphi^{(0)}(r)+\frac{Q(r)}{2}, \quad \varphi^{(0)}(r)=\int_{-1}^{1} \varphi\left(r, \mu^{\prime}\right) d \mu^{\prime} .
\end{gathered}
$$

Уравнение (1) решается в области $G=\{-1 \leqslant \mu \leqslant 1,0 \leqslant r \leqslant R\}$. Граничные условия для уравнения (1) определим в виде

$$
\varphi(R, \mu)=0 \quad \text { при } \quad \mu \leqslant 0 .
$$

В методе Владимирова вводятся новые независимые переменные

$$
x=\mu r, \quad y=r \sqrt{1-\mu^{2}}, \quad r=\sqrt{x^{2}+y^{2}}, \quad \mu=\frac{x}{\sqrt{x^{2}+y^{2}}} .
$$

В переменных $x, y$ уравнение (1) примет вид

$$
\frac{\partial \psi}{\partial x}+\alpha \psi=F
$$

где

$$
\psi(x, y)=\varphi\left(\sqrt{x^{2}+y^{2}}, \frac{x}{\sqrt{x^{2}+y^{2}}}\right) .
$$

В процессе преобразования уравнения (1) к новым переменным область $G$ взаимно однозначно переходит в полукруг $g=\left\{0 \leqslant y \leqslant R, x^{2}+y^{2} \leqslant R\right\}$. При этом линии $r \sqrt{1-\mu^{2}}=$ const, являющиеся характеристиками дифференциальной части 


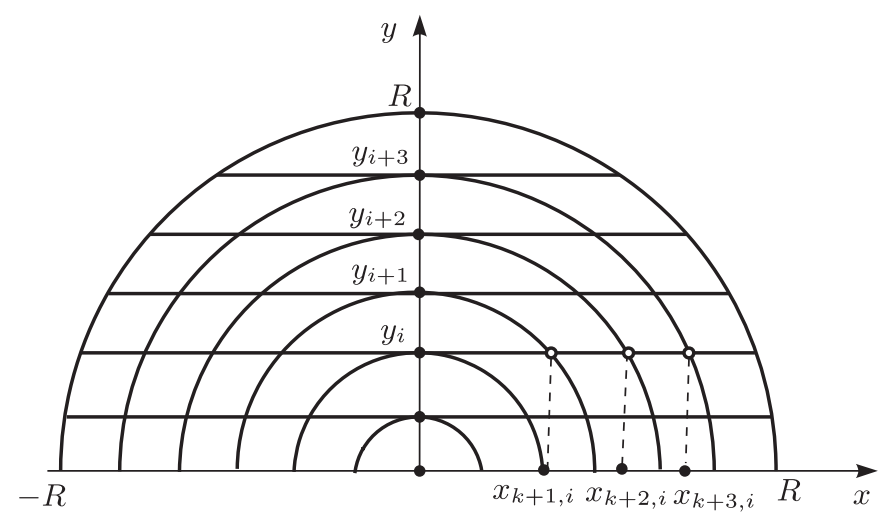

Рис. 1. Расположение узлов сетки.

оператора интегро-дифференциального уравнения (1), перейдут в прямые линии $y=$ const.

Граничные условия для новой функции $\psi(x, y)$ принимают вид

$$
\psi\left(x, \sqrt{R^{2}-x^{2}}\right)=0, \quad-R \leqslant x \leqslant 0, \quad \text { или } \quad \psi\left(-\sqrt{R^{2}-y^{2}}, y\right)=0, \quad 0 \leqslant y \leqslant R .
$$

Таким образом, линейное дифференциальное уравнение (1) в переменных $x, y$ на линиях $y=$ const превращается в обыкновенное дифференциальное уравнение (4). Решая уравнение (4) при условии (5) как обыкновенное дифференциальное уравнение, получим

$$
\psi(x, y)=\int_{-\sqrt{R^{2}-y^{2}}}^{x} F\left(\sqrt{x^{\prime 2}+y^{2}}\right) \exp \left\{-\int_{x^{\prime}}^{x} \alpha\left(\sqrt{x^{\prime \prime 2}+y^{2}}\right) d x^{\prime \prime}\right\} d x^{\prime} .
$$

Для численного интегрирования уравнения (4) разобьем область определения решения $g$ линиями $r=$ const и $y=$ const на интервалы (рис. 1). Индекс $i$ обозначает порядковый номер координатной линии $y=$ const, индекс $k$ - порядковый номер точки на каждой из линий $y=$ const.

Вычисление функции $\psi(x, y)=\varphi(r, \mu)$ производится в узловых точках с координатами

$$
x=x_{k, i}= \pm \sqrt{r_{k}^{2}-r_{i}^{2}}, \quad y=y_{i}=r_{i},
$$

где $i=0,1, \ldots, p$ - число точек по радиусу, $k= \pm i, \pm(i+1), \ldots, \pm p$. В результате на каждой линии $y=y_{i}$ проводим последовательное интегрирование уравнения (4) по $x$. Для интервала $\left(x_{k+1, i}, x_{k+2, i}\right)$ получим

$$
\begin{aligned}
\psi_{k+2, i}= & \psi_{k+1, i} \exp \left\{-\int_{x_{k+1, i}}^{x_{k+2, i}} \alpha\left(\sqrt{x^{\prime \prime 2}+y_{i}^{2}}\right) d x^{\prime \prime}\right\}+ \\
& +\int_{x_{k+1, i}}^{x_{k+2, i}} F\left(\sqrt{x^{\prime 2}+y_{i}^{2}}\right) \exp \left\{-\int_{x^{\prime}}^{x_{k+2, i}} \alpha\left(\sqrt{x^{\prime \prime 2}+y_{i}^{2}}\right) d x^{\prime \prime}\right\} d x^{\prime} .
\end{aligned}
$$

29 июня 1953 года В. С. Владимиров защитил диссертацию на соискание ученой степени кандидата физико-математических наук на тему "Численное решение кинетического уравнения для сферы" [5]. 
“Следует отметить, что примерно в это же время в Лос-Аламосской национальной лаборатории США был разработан похожий метод Карлсона. Разница в методах следующая: в методе Карлсона численное интегрирование проводится по радиусу при фиксированных косинусах угла между радиусом и направлением движения частицы, в то время как в методе Владимирова - по характеристикам, т. е. по путям (по кусочно-прямым линиям), по которым движутся частицы..." [5].

Метод Владимирова послужил основой для создания дивергентного варианта метода характеристик - метода характеристических трубок [6]-[9]. Достоинством характеристических методов [7]-[9] является возможность построения разностных схем, обладающих свойством монотонности.

В 1954 году для решения многослойного уравнения диффузии В. С. Владимиров предложил метод факторизации [3], [10], [11]. Сущность метода факторизации состоит в том, что дифференциальный оператор второго порядка разлагается на два линейных множителя (дифференциальные операторы первого порядка) с помощью неизвестной функции, удовлетворяющей уравнению Риккати. Для данной задачи решение уравнения Риккати положительно, и поэтому численное интегрирование одного из уравнений первого порядка при возрастании радиуса устойчиво, а второго уравнения первого порядка также устойчиво, но при уменьшении радиуса.

Рассмотрим краевую задачу

$$
\begin{gathered}
-\left(p u^{\prime}\right)^{\prime}+q u=f(r), \quad 0<r<R ; \\
h u(0)-u^{\prime}(0)=0, \quad u(R)+H u^{\prime}(R)=0 .
\end{gathered}
$$

Для этой задачи дифференциальный оператор факторизуется в виде двух линейных операторов первого порядка,

$$
-\frac{d}{d r}\left(p \frac{d u}{d r}\right)+q u=-\left(\frac{d}{d r}+\frac{g}{p}\right)\left(p \frac{d}{d r}-g\right) u,
$$

причем вспомогательная функция $g(r)$ удовлетворяет уравнению Риккати

$$
g^{\prime}+\frac{g^{2}}{p}=q, \quad g(0)=p(0) h .
$$

В результате задача (6), (7) сводится к линейной системе краевых задач

$$
\begin{gathered}
y^{\prime}+\frac{g}{p} y=f, \quad y(0)=0 \\
-p u^{\prime}+g u=y, \quad u(R)=\frac{H y(R)}{p(R)+H g(R)} .
\end{gathered}
$$

Решение краевой задачи (8) для уравнения Риккати положительно $(p(0) h>0)$. Это обеспечивает устойчивость численного решения уравнения (9) при возрастании $r$ от 0 до $R$ и уравнения (10) при убывании $r$ от $R$ до 0.

“Изложенный метод факторизации эквивалентен методу прогонки с той только разницей, что в методе факторизации сначала факторизуется дифференциальный оператор и полученные устойчивые уравнения решаются численными методами, в то время как в методе прогонки сначала применяются к исходному уравнению трехточечные разностные схемы и далее проводится факторизация соответствующего разностного оператора" [5]. 
Осенью 1954 года методом факторизации в отделе В. С. Владимирова по заданию А. Д. Сахарова была рассчитана серия задач по обоснованию работы изделия РДС-37.

В своих "Воспоминаниях", по-видимому имея в виду методы факторизации и прогонки, А. Д. Сахаров написал следующее [12]: "Но, конечно, для расчета изделий, основанных на "третьей идее", недостаточно было анализа отдельных процессов в упрощающих предположениях - нужны были новые методики сложных численных вычислений, пригодные для ЭВМ. Такие методики были разработаны математиками объекта и московских математических групп".

Помимо практических задач В. С. Владимиров большое внимание уделял теоретическим исследованиям уравнения переноса. Им были доказаны теоремы существования и единственности решения уравнения переноса, установлены свойства собственных значений и собственной функции, исследованы особенности производных в окрестности раздела двух сред, предложен новый вариационный принцип [3], [13]. Разработанный в 1954 году, этот принцип в мировой литературе часто называют вариационным принципом Владимирова [14].

Рассмотрим краевую задачу для односкоростного стационарного уравнения пеpeноса

$$
(s, \nabla \varphi)+\alpha(x) \varphi=\frac{1}{4 \pi} \int_{\Omega} \theta(x, \mu) \varphi\left(x, s^{\prime}\right) d s^{\prime}+Q(x, s), \quad x=\left(x_{1}, x_{2}, x_{3}\right) \in D,
$$

с граничным условием

$$
\left.\varphi\right|_{\Gamma}=0, \quad(s, n) \leqslant 0,
$$

где $\Gamma$ - граница выпуклой области $D, n$ - вектор внешней нормали к $\Gamma, \Omega$ - единичная сфера, $\mu=\left(s, s^{\prime}\right)$. Пусть индикатриса $\theta(x, \mu)$ и источник $Q(x, s)$ - четные функции относительно $\mu$ и $s$ соответственно. Для новой неизвестной функции

$$
\Psi(x, s)=\frac{1}{2}[\varphi(x, s)+\varphi(x,-s)]
$$

задача (11), (12) эквивалентна самосопряженной задаче

$$
\begin{gathered}
-\left(s, \nabla\left(s, \frac{1}{\alpha} \nabla \Psi\right)\right)+\alpha \Psi=\frac{1}{4 \pi} \int_{\Omega} \theta(x, \mu) \Psi\left(x, s^{\prime}\right) d s^{\prime}+Q, \\
\Psi-\left.\frac{1}{\alpha}(s, \nabla \Psi)\right|_{\Gamma}=0, \quad(s, n) \leqslant 0, \\
\Psi+\left.\frac{1}{\alpha}(s, \nabla \Psi)\right|_{\Gamma}=0, \quad(s, n) \geqslant 0,
\end{gathered}
$$

Для полученной новой задачи (13) строится вариационный принцип и выделяются естественные граничные условия. Соответствующая билинейная форма имеем довольно простую структуру, что позволяет эффективно применять прямые методы вариационного исчисления к исследованию и приближенному решению исходной задачи.

В 1952 году В. С. Владимиров и Н. Н. Боголюбов начали обсуждение вопроса о применении метода Монте-Карло к решению многомерных задач теории переноса частиц в связи со следующей задачей. Около восьми месяцев длилась разработка 
методики для численного решения уравнения Пайерлса для области с цилиндрической симметрией. Для решения задачи объем цилиндра разбивали на элементарные области - торы, осевые сечения которых представляют четырехугольники. Интегральное уравнение заменяли системой алгебраических уравнений. Основными этапами этой работы были вывод формул для элементов матрицы, их расчет и итерирование матрицы 47-го порядка. В 1956 году в первом номере нового журнала "Теория вероятностей и ее применения" В. С. Владимиров опубликовал первую в СССР работу по методу Монте-Карло.

В январе 1955 года В.С. Владимиров покинул объект в связи с переводом на работу в ЦНИИ-58 Министерства оборонной промышленности СССР. В ЦНИИ-58 группа под руководством В. С. Владимирова проводила расчеты задач нейтронной физики для заряда РДС-41. Испытание атомного заряда РДС-41 прошло успешно 16 марта 1956 года на Семипалатинском полигоне.

В 1956 году В. С. Владимиров перешел на должность старшего научного сотрудника в Отдел теоретической физики Математического института им. В. А. Стеклова (МИАН). Вот как описал окончание своего участия в атомном проекте В. С. Владимиров в книге "Я - сын трудового народа" [5]: “Теперь я считал свою работу над атомной проблемой законченной и поставил вопрос о возвращении меня в Академию наук. Мое прямое военное служение Родине закончилось в 33 года".

\section{Список литературы}

[1] В. С. Владимиров, Вычисл. матем., 3 (1958), 3-33.

[2] Г. И. Марчук, Методы расчета ядерных реакторов, Атомиздат, М., 1961.

[3] В. С. Владимиров, Вопросы атомной науки и техники. Серия: Математическое моделирование физических прочессов, 4 (1992), 105-109.

[4] В. А. Чуянов, "Владимирова метод”, Математическая энииклопедия, т. I, Советская энциклопедия, М., 1977.

[5] В. С. Владимиров, Я - сын трудового народа, ФАЗИС, М., 2007.

[6] А. В. Никифорова, В. А. Тарасов, В.Е. Трощиев, Ж. вычисл. матем. и матем. физ., 12:4 (1972), 1041-1048.

[7] В. Е. Трощиев, А. В. Нифанова, Ю. В. Трощиев, Докл. РАН, 394:4 (2004), 454-458.

[8] В. Е. Трощиев, А. В. Нифанова, Математическое моделирование, 18:7 (2006), 24-42.

[9] А. В. Нифанова, Характеристические $S_{n}$-методы для кинетического уравнения переноса нейтронов в сферических системах, Дис. ... канд. физ.-матем. наук., ТРИНИТИ, Троицк, 2008.

[10] В. С. Владимиров, Прикладн. матем. и механ., 19:3 (1955), 315-324.

[11] В. С. Владимиров, Вопросы атомной науки и техники. Серия: Математическое моделирование физических процессов, 1 (2002), 6-7.

[12] А. Д. Сахаров, Воспоминания, т. 1, Права человека, М., 1996.

[13] В. С. Владимиров, Математические задачи односкоростной теории переноса частич, Тр. МИАН СССР, 61, Изд-во АН СССР, М., 1961.

[14] Ю.Н. Дрожжинов, "Владимирова вариационный принцип”, Математическая энциклопедия, т. 1, Советская энциклопедия, М., 1977. 